\title{
TANGENTIAL LIMITS OF STARLIKE UNIVALENT FUNCTIONS
}

\author{
J. B. TWOMEY
}

ABSTRACT. Let $f$ be starlike univalent in the unit disc, let $\gamma>1$ and let $K>$ 0 . Then $f(z)$ tends to a limit as $z \rightarrow e^{i \theta}$ inside $\left\{z:\left|e^{i \theta}-z\right| \leq K(1-|z|)^{1 / \gamma}\right\}$ for every $\theta$ in $[0,2 \pi]$. This result is sharp.

1. Introduction. We begin by defining certain subsets $\Omega$ of the unit disc $U=$ $\{z:|z|<1\}$ which make tangential contact with the boundary $\partial U$ of the disc. Let $\phi$ be a decreasing continuous function on $[0,1]$ with $\phi(1)=0$, for which

$$
\frac{1-r}{\phi(r)} \rightarrow 0 \quad \text { as } r \rightarrow 1
$$

Let $K>0, \theta \in[0,2 \pi]$ and set

$$
\Omega(\phi, \theta, K)=\left\{z \in U:\left|e^{i \theta}-z\right| \leq K \phi(r)\right\},
$$

where $r=|z|$. The region $\Omega$ makes tangential contact with $\partial U$ at $e^{i \theta}$; when $\phi(r)=\left(1-r^{2}\right)^{1 / 2}$, for instance, $\Omega(\phi, \theta, 1)$ is the disc with radius $\frac{1}{2}$ and centre $\frac{1}{2} e^{i \theta}$. We next note the following recent result of Nagel, Rudin and Shapiro for functions in the Dirichlet space $D$, that is, the space of functions analytic in $U$ for which

$$
\iint_{U}\left|f^{\prime}(z)\right|^{2} d x d y
$$

is finite.

ThEOREM A [7]. For $0<r<1$, set

$$
\phi(r)=\left(\log \frac{1}{1-r}\right)^{-1}
$$

and let $f \in D$. Then, for every $K>0, \lim f(z)$ exists finitely as $z \rightarrow e^{i \theta}$ within $\Omega(\phi, \theta, K)$ for almost all $\theta$ in $[0,2 \pi]$.

The class $D$ contains all bounded univalent functions since, if $f$ is univalent (i.e. analytic and one-to-one in $U$ ), the integral (1.1) represents the area of $f(U)$. A standard argument now shows that the conclusion of Theorem A holds for all univalent functions. For if $f$ is univalent in $U, f(U)$ cannot be the entire complex plane, so there is a point $w_{0}$ in the complement of $f(U)$ and then, by a simple argument [10, pp. 302-303], there is a number $a$ such that

$$
g(z)=\left[\left(f(z)-w_{0}\right)^{1 / 2}+a\right]^{-1}
$$

Received by the editors March 5, 1984 and, in revised form, April 29, 1985.

1980 Mathematics Subject Classification. Primary 30C55, 30C45, 30D40.

Key words and phrases. Tangential limits, univalent functions, starlike functions. 
is univalent and bounded in $U$. Then

$$
f(z)=w_{0}+\left\{\frac{1}{g(z)}-a\right\}^{2}, \quad z \in U
$$

and so, if $g$ tends to a finite limit as $z \rightarrow e^{i \theta}$ within $\Omega(\phi, \theta, K), f$ does also, unless the limit for $g$ is zero. By a uniqueness theorem of F. and M. Riesz, this can happen on $\partial U$ only at a set of measure zero $[\mathbf{5}, \mathrm{p} .76]$. The conclusion of Theorem A thus holds for all univalent functions, as asserted.

Beurling $[\mathbf{1}]$ has shown that the exceptional set of points on $\partial U$ at which a function in $D$ fails to have a finite angular limit is a set of logarithmic capacity zero and in [7] further theorems are derived which 'interpolate' between this result and Theorem A. Nagel et al. show, in fact, that for certain other kinds of exceptional sets $E$-intermediate between sets of logarithmic capacity zero and measure zeroand $f \in D, \lim f(z)$ exists as $z \rightarrow e^{i \theta}$ within $\Omega(\phi, \theta, K)$ for every $e^{i \theta} \in \partial U \backslash E$ where, this time, $\phi(r)=(1-r)^{\varepsilon}, 0<\varepsilon<1$, and $\varepsilon$ depends only on the size of the exceptional set $E$. (See $[7]$ for details.) All such results extend to the full class of univalent functions.

In this note we are concerned mainly with the boundary behaviour of starlike univalent functions. It is well known that such functions have radial (and hence angular) limits, finite or infinite, at all points on $\partial U$ (see, for example, [4]), but we strengthen this result by showing that starlike univalent functions have certain fixed tangential limits at all points on $\partial U$. We further show that our result is, in a certain sense, best possible.

2. Statement of results. We say a function $f: U \rightarrow \mathbf{C}$ has a tangential $T_{\gamma}$-limit at a point $e^{i \theta}$ on $\partial U$ for $\gamma>1$ if there exists $l \in \mathbf{C} \cup\{\infty\}$ such that, for every $K>0$,

$$
f(z) \rightarrow l \quad \text { as } z \rightarrow e^{i \theta}, \quad z \in \Omega(\phi, \theta, K),
$$

where $\phi(r)=(1-r)^{1 / \gamma}, 0 \leq r<1$. A function $f$, univalent in $U$, with $f(0)=0$, is said to be starlike univalent if $f(U)$ is starshaped with respect to 0 , that is, $f(U)$ contains the line segment $[0, w]$ whenever it contains $w$. We can now state our first theorem.

THEOREM 1. Let $f$ be starlike univalent in $U$. Then $f$ has a (finite or infinite) $T_{\gamma}$-limit for every $\gamma>1$ at each fixed point on $\partial U$.

For starlike univalent functions, therefore, the existence of a radial limit at a point implies the existence of all $T_{\gamma}$-limits, $\gamma>1$, at that point, and the question arises whether this result holds generally for all univalent functions. The answer, in fact, is known to be negative, even for the subclass $C_{i}$ of univalent functions $f$ for which $f(U)$ is convex in the direction of the imaginary axis. (A domain $G$ is convex in the direction of the imaginary axis if each line parallel to the imaginary axis intersects $G$ in one interval or not at all.) Indeed Gaier and Pommerenke [3] have shown that if $\left(z_{n}\right)$ is any sequence of points in $U$ such that $z_{n} \rightarrow 1$ and $\arg \left(z_{n}-1\right) \rightarrow \pi / 2(n \rightarrow \infty)$, then there exists a bounded function $f \in C_{i}$ for which the radial limit at $z=1$ exists but $f\left(z_{n}\right)$ diverges.

Our second theorem shows that the conclusion of Theorem 1 is best possible as regards the nature of the tangential limit that exists at each point on $\partial U$. 
THEOREM 2. Let $\eta$ be a positive function defined on $[0,1)$ for which $\eta(r) \rightarrow 0$ and $\eta(r) \log (1 /(1-r)) \rightarrow \infty$ as $r \rightarrow 1^{-}$, and set

$$
\psi(r)=(1-r)^{\eta(r)}, \quad 0 \leq r<1 .
$$

Let the arc $\Gamma:[0,1) \rightarrow U$ be defined by

$$
\Gamma(r)=r e^{i \psi(r)}, \quad 0 \leq r<1 .
$$

Then there exists a bounded starlike function $f$ which does not tend to a limit as $z \rightarrow 1$ along $\Gamma$.

It is a consequence of Theorem $\mathrm{A}$, of course, that, for starlike univalent functions, $\lim f(z)$ exists as $z \rightarrow e^{i \theta}$ within $\Omega(\phi, \theta, K)$, with $\phi(r)=[-\log (1-r)]^{-1}$, at all points $e^{i \theta}$ on $\partial U$ except possibly for a set of $\theta$ of measure zero. A further sharpening of the conclusion of Theorem 1 is possible outside a set which is topologically small. Indeed if $f$ is starlike univalent, then, for all $e^{i \theta} \in \partial U$ except possibly for a set of $\theta$ of first category, $\lim f(z)$ exists as $z \rightarrow e^{i \theta}$ in any way from inside $U$; this follows from the Collingwood maximality theorem $[\mathbf{2}$, p. 76] and the fact that starlike univalent functions have radial limits at all points on $\partial U$. Conversely, given any set $E \subset \partial U$ of type $F_{\sigma}$ and of first category, there exists a bounded starlike function $f$ such that $\partial U \backslash E$ is precisely the set of points at which $f$ possesses unrestricted limits. This last result is easily established using the examples in [6, pp. 5-7].

3. Proof of Theorem 1. Let $f$ be starlike univalent in $U$ and suppose, without loss of generality, that $f^{\prime}(0)=1$. Then, by the representation formula for starlike univalent functions $[\mathbf{9}, \mathrm{p} .43]$,

$$
f(z)=z \exp \left\{\frac{1}{\pi} \int_{0}^{2 \pi} \log \frac{1}{1-e^{-i t} z} d F(t)\right\}, \quad z \in U,
$$

where $F$ is increasing on $(-\infty, \infty)$ and $F(t)-t$ is periodic with period $2 \pi$. If we normalise $F$ to satisfy the condition

$$
\int_{0}^{2 \pi}[F(t)-t] d t=0
$$

then it follows easily from (3.1) (see [8, p. 210] for details) that

$$
\arg f\left(r e^{i \theta}\right)=\theta+\frac{1}{2 \pi} \int_{0}^{2 \pi} \frac{\left(1-r^{2}\right)[F(t)-t]}{1-2 r \cos (\theta-t)+r^{2}} d t,
$$

for $0 \leq r<1$.

We now let $\theta_{0} \in[0,2 \pi]$ and consider first the case where $f\left(r e^{i \theta_{0}}\right)=O(1)$ as $r \rightarrow 1$. Then $F$ is continuous at $\theta_{0}[\mathbf{4}]$ and it follows from (3.2), by a classical result $[5$, p. 11], that

$$
\arg f(z) \rightarrow F\left(\theta_{0}\right)
$$

as $z \rightarrow z_{0}=e^{i \theta_{0}}$ in any way from inside $U$. To complete the proof that $f$ has a finite $T_{\gamma}$-limit at $z_{0}$ for every $\gamma>1$, it is enough to show that, for each $\gamma>1$, $|f(z)|$ tends to a finite limit as $z \rightarrow z_{0}$ inside $\Omega=\Omega\left(\phi, \theta_{0}, 1\right)$ where, for $0<r<1$, $\phi(r)=(1-r)^{\varepsilon}$, and $\varepsilon=1 / \gamma$. First, by $(3.1)$,

$$
\log \left|f\left(r e^{i \theta}\right)\right|=\frac{1}{2 \pi} \int_{0}^{2 \pi} l(r, \theta-t) d F(t),
$$


where $l(r, x)=\log \left\{r /\left|1-r e^{i x}\right|^{2}\right\}$. Now $l(r, x)$ is an increasing function of $r$ in $(0,1)$ for each fixed $x, l(r, x) \geq l(r, \pi)$ for $x \in[0,2 \pi]$, and $f\left(r z_{0}\right)=O(1)$ as $r \rightarrow 1$, so we deduce from (3.3) and the monotone convergence theorem for integrals that

$$
\log \left|f\left(z_{0}\right)\right|=\lim _{r \rightarrow 1} \log \left|f\left(r z_{0}\right)\right|=\frac{1}{\pi} \int_{0}^{2 \pi} \log \frac{1}{2\left|\sin \left(\frac{1}{2}\left(\theta_{0}-t\right)\right)\right|} d F(t)
$$

is finite. Next we note that, by (3.3), for $z=r e^{i \theta} \in U$,

$$
\log \left\{\left|\frac{f\left(r z_{0}\right)}{r}\right|^{\varepsilon}\left|\frac{z}{f(z)}\right|\right\}=\frac{1}{\pi} \int_{0}^{2 \pi} \log \frac{\left|1-r e^{i(\theta-t)}\right|}{\mid 1-r e^{i\left(\theta_{0}-t\right) \mid \varepsilon}} d F(t) .
$$

Now if $x, y$ are real numbers satisfying $\left|e^{i x}-r e^{i y}\right| \leq(1-r)^{\varepsilon}$, then

$$
\left|1-r e^{i x}\right| \leq 3\left|1-r e^{i y}\right|^{\varepsilon}, \quad 0 \leq r<1 \text {. }
$$

For

$$
\begin{aligned}
2 r^{1 / 2}|\sin (x / 2)| & \leq 2 r^{1 / 2}|\sin (y / 2)|+2 r^{1 / 2}|\sin ((x-y) / 2)| \\
& \leq\left|1-r e^{i y}\right|+(1-r)^{\varepsilon} \leq 2\left|1-r e^{i y}\right|^{\varepsilon}
\end{aligned}
$$

and so

$$
\left|1-r e^{i x}\right|^{2}=(1-r)^{2}+4 r \sin ^{2}(x / 2) \leq 5\left|1-r e^{i y}\right|^{2 \varepsilon},
$$

which clearly gives (3.6). Hence, if $z=r e^{i \theta} \in \Omega$, by (3.6), the integrand on the right of (3.5) is bounded above by $\log 3$ and below by

$$
\log \left\{3^{-\gamma}\left|1-r e^{i\left(\theta_{0}-t\right)}\right|^{\gamma-\varepsilon}\right\} \geq-\gamma \log 3-(\gamma-\varepsilon) \log \frac{1}{\left|\sin \left(\frac{1}{2}\left(\theta_{0}-t\right)\right)\right|}
$$

when $r \geq 1 / 4$. By the finiteness of the integral in (3.4) and the dominated convergence theorem for integrals, it thus follows from (3.5) that, as $z \rightarrow z_{0}$ in $\Omega$,

$$
\log \left\{\left|\frac{f\left(r z_{0}\right)}{r}\right|^{\varepsilon}\left|\frac{z}{f(z)}\right|\right\} \rightarrow \frac{1}{\pi} \int_{0}^{2 \pi} \log \left\{\left|1-e^{i\left(\theta_{0}-t\right)}\right|^{1-\varepsilon}\right\} d F(t),
$$

that is, by (3.4),

$$
\varepsilon \log \left|f\left(z_{0}\right)\right|-\log |f(z)| \rightarrow(\varepsilon-1) \log \left|f\left(z_{0}\right)\right| .
$$

Hence $|f(z)| \rightarrow\left|f\left(z_{0}\right)\right|$ as $z \rightarrow z_{0}$ in $\Omega$, as required.

Suppose finally that $f\left(r z_{0}\right) \neq O(1), z_{0}=e^{i \theta_{0}}$, as $r \rightarrow 1$ for $\theta_{0} \in[0,2 \pi]$, so that $\left|f\left(r z_{0}\right)\right| \rightarrow \infty(r \rightarrow 1)$ by monotonicity. Let $\varepsilon, \phi, \Omega$ be as above and suppose that $z=r e^{i \theta} \in \Omega$. Then, by (3.6), the integral on the right of (3.5) is bounded above by $2 \log 3$ and so

$$
\left|f\left(r z_{0}\right) / r\right|^{\varepsilon} \leq 9|f(z) / z|
$$

Hence $f(z) \rightarrow \infty$ as $z \rightarrow z_{0}$ in $\Omega$ and the proof of Theorem 1 is complete.

4. Proof of Theorem 2. The examples we use to prove Theorem 2 are simple adaptations of examples constructed in [6] and involve functions of the form

$$
\begin{gathered}
g(z)=z+\sum_{1}^{\infty} z_{n} g_{n}(z) \\
g_{n}(z)=1-\left(1-z / z_{n}\right)^{\alpha_{n}} \quad(n \geq 1, z \in U)
\end{gathered}
$$


where the $z_{n}$ are points on $\partial U,\left(\alpha_{n}\right)$ is a sequence of numbers in $(0,1)$, and the branch in (4.2) is chosen so that $g_{n}(0)=0$. A function of this type maps $U$ onto a domain consisting roughly of the disc $U$ with narrow tooth-like extensions, of approximate length 1 , radiating outwards from the points $z_{n}$. For use below we note here that elementary calculations show there is a positive absolute constant $B$ such that, if $0<\delta_{n}<\frac{1}{2}$,

$$
\left|g_{n}(z)\right| \leq-B \alpha_{n} \log \delta_{n} \text { for }\left|z-z_{n}\right| \geq \delta_{n}
$$

To construct the specific examples we require, we let the functions $\eta$ and $\psi$ be as in Theorem 2 and choose a sequence $\left(r_{n}\right)$ of numbers in $(5 / 6,1)$, increasing to 1 , such that

$$
B \eta\left(r_{n}\right) \geq 6^{-n-1} \quad(n \geq 1)
$$

and

$$
1 / 4 \geq \theta_{n}=\psi\left(r_{n}\right) \geq 2 \theta_{n+1} \quad(n \geq 1) .
$$

For $n \geq 1$, let $\alpha_{n}$ be defined by

$$
\left(1-r_{n}\right)^{\alpha_{n}}=1 / 6
$$

and let $\Delta_{n}$ denote the closed disc centered at $z_{n}=e^{i \theta_{n}}$ with radius $\delta_{n}=\theta_{n} / 4$. With $\left(\alpha_{n}\right)$ and $\left(z_{n}\right)$ thus defined, we now suppose that $g$ is defined by (4.1) and (4.2). Then, by (4.3), (4.6) and (4.4), for $z \in \bar{U} \backslash \Delta_{n}$ and $n \geq 1$,

$$
\left|g_{n}(z)\right| \leq 6 B \eta\left(r_{n}\right) \leq 6^{-n}
$$

and so the series in (4.1) is uniformly bounded on $U$ and converges uniformly on compact subsets of $U$. Hence $g$ is analytic and bounded on $U$.

Now let $\Gamma$ be the curve defined in Theorem 2. Then since the closed discs $\Delta_{n}$ are disjoint, by (4.5), there are points on $\Gamma$, arbitrarily close to 1 , which lie in none of the discs $\Delta_{n}$. At such points, by (4.1) and (4.7),

$$
|g(z)| \leq 1+\sum_{1}^{\infty} 6^{-n}=\frac{6}{5} .
$$

On the other hand, for $n \geq 1, r_{n} z_{n}$ also lies on $\Gamma$ and, by (4.1), (4.6) and (4.7),

$$
\begin{aligned}
\left|g\left(r_{n} z_{n}\right)\right| & \geq r_{n}+1-\frac{1}{6}-\sum_{k \neq n}\left|g_{k}\left(r_{n} z_{n}\right)\right| \\
& \geq \frac{5}{3}-\sum_{1}^{\infty} 6^{-n}=\frac{5}{3}-\frac{1}{5}>\frac{7}{5}
\end{aligned}
$$

Hence $g$ does not tend to a limit as $z \rightarrow 1$ along $\Gamma$.

It is easily verified that $\operatorname{Re} g^{\prime}(z)>0(z \in U)$ and so $g$ is univalent in $U$, but rather than attempt to prove that $g$ is starlike univalent in $U$, we show instead that it is a simple matter to construct from $g$ a starlike function $f$ with the properties we require. For this purpose we normalise (if necessary) the function $g$ so that it satisfies $g^{\prime}(0)=1$ and set

$$
f(z)=z \exp \left\{\int_{0}^{z} \frac{g^{\prime}(w)-1}{w} d w\right\}, \quad z \in U
$$


Then $f(0)=0$ and

$$
\operatorname{Re}\left(z f^{\prime}(z) / f(z)\right)=\operatorname{Re} g^{\prime}(z)>0 \quad(z \in U),
$$

so $[9$, p. 42] $f$ is starlike univalent in $U$. Taking logarithms in (4.8), and integrating by parts, we obtain

$$
\log \frac{f(z)}{z}=\frac{g(z)-z}{z}+\int_{0}^{z} \frac{g(w)-w}{w^{2}} d w .
$$

The second term on the right of (4.9) has a bounded derivative in $U$ and consequently has a continuous extension to the closed disc $\bar{U}$. Hence, by (4.9), $f$ is bounded in $U$ and does not tend to a limit as $z \rightarrow 1$ along $\Gamma$, since $g$ does not. This completes the proof of Theorem 2 .

I wish to thank the referee for drawing my attention to reference [3].

\section{REFERENCES}

1. A. Beurling, Ensembles exceptionnels, Acta Math. 72 (1940), 1-13.

2. E. F. Collingwood and A. J. Lohwater, The theory of cluster sets, Cambridge Univ. Press, London, 1966.

3. D. Gaier and Ch. Pommerenke, On the boundary behavior of conformal maps, Michigan Math. J. 14 (1967), 79-82.

4. F. R. Keogh, Some theorems on conformal mapping of bounded starshaped domains, Proc. London Math. Soc. (3) 9 (1959), 481-491.

5. P. Koosis, Introduction to $H_{p}$ spaces, London Math. Soc. Lecture Note Ser., Vol. 40, Cambridge Univ. Press, London, 1980.

6. A. J. Lohwater and G. Piranian, The boundary behavior of functions analytic in a disk, Ann. Acad. Sci. Fenn. Ser. A I Math. Dissertationes 239 (1957), 1-17.

7. A. Nagel, W. Rudin and J. H. Shapiro, Tangential boundary behavior of functions in Dirichlet-type spaces, Ann. of Math. (2) 116 (1982), 331-360.

8. Ch. Pommerenke, On starlike and convex functions, J. London Math. Soc. 37 (1962), 209-224.

9. __ Univalent functions, Vandenhoeck \& Ruprecht, Göttingen, 1975.

10. W. Rudin, Real and complex analysis, 2nd ed., McGraw-Hill, New York, 1974.

Department of Mathematics, University College, Cork, Ireland 